\title{
Comment
}

DO WE KNOW THE VALUE OF WHAT WE ARE DOING?

\section{The right weight: good practice in evaluating science communication}

\section{Giuseppe Pellegrini}

\begin{abstract}
Evaluations of science communication activities before, during and after their implementation can provide findings that are useful in planning further activities. As some selected examples show, designing such evaluation is complex: they may involve assessment at various points, a mix of quantitative and qualitative methods, and show that impacts differ when seen from different perspectives.
\end{abstract}

Social researchers, scientists and communicators have all in recent years stressed the need to carry out assessment of science communication processes. Many institutions that organise festivals, exhibition, visits and public information campaigns have launched initiatives to test the effectiveness of their processes of public communication of science. But what are the elements that make an effective evaluation? And how can you develop tools, processes and practices to achieve efficiently a correct assessment? I will propose some answers to these questions analysing significant examples of satisfactory and credible assessment.

One of the main goals of a communication assessment is to eliminate or replace the actions that generate undesirable effects when performing a communication activity. For this reason, the evaluation focus is often on what is working, and what is not, in the ongoing activity. This is commonly known as formative assessment; it requires analysis of patterns of interaction among the actors and monitoring of the use to which the available resources are put.

An example of effective formative assessment in communication through the web can be found in a study of the integration of technology in university coursework [1]. A semester-long wiki project was planned and evaluated using a formative assessment plan. The assessment indicated future structural and communicative modifications, taking into account students' suggestions. An open-ended questionnaire was used to verify 'the effectiveness of producing a better understanding of real-world applications through the wiki project and their perception of the wiki project's shortcomings and/or challenges".

Evaluation can also be effective at the end of the communicative process (summative evaluation) if the aims and the assessment design are aligned, giving the opportunity to 
monitor differences before and after the communication intervention. An instrument to test scientists' writing skills in public communication of science was used as a baseline survey in order to evaluate written communication within an analytical framework [2]. The study contributed to a better understanding of scientists' communication skills, revealing that to some extent they need to learn a new language of science, "the discourse of public communication of science", as a means of engaging with the public.

When you prepare a science communication activity and you want to check the intended changes, there is a strong temptation to adopt standard survey instruments that are easy-to-use and have a low impact on respondents. This is a typical reduction of complexity; the standard tools for assessment tend to be based on a narrow selection of information.

In many cases it is possible to mixed quantitative and qualitative measurements in order to achieve a complete frame of results and effects, also for small-scale initiatives such as may be organised in informal contexts. This was the case of the Discovery07, a Dutch science party, which had the main goal to verify a change in public opinion on the image of science and scientists through a "party with a meaning". The initiative included live music, live scientific research activities and other presentations made by young scientists.

The evaluation followed a before-and-after procedure for collecting data in order to test the effect of the party activities. In addition, face-to-face interviews were carried out with participants during the event. The respondents' statements provide an interesting frame of insights to contextualize quantitative findings [3].

Efforts to improve evaluation in public communication of science and technology have extended across multiple facets of the process. Evaluation may have to take into account results and outcomes that are quite different from each other.

Results can be seen as products of what a particular communication programme envisaged, privileging the point of view of the promoters. Outcomes, however, are viewed as changes produced by the communication with a broad focus on all actors involved in the process. This means that it is possible to have different judgments in the two cases, so that good results offer no guarantees in terms of outcomes.

An interesting case of mixing results and outcomes assessment was proposed for the public campaign, Evaluating My Plate, a United States Department of Agriculture initiative for better nutrition [4]. This campaign had the main goal "to support Americans in building healthy diet" bridging the gap between knowledge and behaviour. The evaluation design considered "qualitative and quantitative research methods to measure both communication implementation and outcomes" [5].

Using a set of variables to collect data, evaluators can identify specific links between communication results and "desired outcomes in both audience knowledge and dietary behaviors". Different factors form the framework proposed as "a rubric to provide a common set of variables to use in the design, collection, analysis, and application of findings" [6]. By following this procedure, researchers should be able to measure effects at various stages and to what extent desired outcomes are achieved. 
An evaluation plan for a science communication activity is based on two main questions: What you want to know? How will you know it? These questions allow us to identify the changes that an activity produces over time. In this way you can select the most sensitive indicators to verify the results and effects of a communication programme. You can then find that the assessment is a circular process that allows you to start with some goals, check them with the help of the actors involved, collecting their different views, and return to plan new activities thanks to the lessons learned with the evaluation. Effective assessment therefore requires the ability to control this circular process, assigning proper weight to each element.

\section{References}

[1] S.W. Bowman (2013), "A formative evaluation of WIKI's as a learning tool in a face to face juvenile justice course", Educational Technology Research and Development 61(1): 3-24.

[2] A. Baram-Tsabari and B.V. Lewenstein (2013), “An Instrument for Assessing Scientists' Written Skills in Public Communication of Science”, Sci. Commun. 35(1): 56-85.

[3] C.M. Koolstra (2008), "An example of a science communication evaluation study: Discovery07, a Dutch science party”, JCOM 7(2): A03.

[4] United States Department of Agriculture, Center for Nutrition Policy and Promotion, MyPlate Background, http://www.cnpp.usda.gov/Publications/MyPlate/Backgrounder.pdf, accessed 4 February 2014

[5] E. Levine, J. Abbatangelo-Gray, A.R. Mobley, G.R. McLaughling and J. Herzog (2012), "Evaluating MyPlate: An Expanded Framework Using Traditional and Nontraditional Metrics for Assessing Health Communication Campaigns", J. Nutr. Educ. Behav. 44: S2-S12.

[6] E.A. Ostrom (2009), "General framework for analyzing sustainability of social-ecological systems", Science 325: 419-422.

\section{Author}

Giuseppe Pellegrini teaches social research methodology at the University of Padova, Italy, and is co-ordinator of the research area Science and Citizens at Observa Science in Society research centre, in Vicenza. His research focuses on social policy, citizenship rights and public participation with specific regard to food issues.

E-mail: giuseppe.pelegrini@unipd.it.

How to CITE: G. Pellegrini, The right weight: good practice in evaluating science communication, JCOM 13(01)(2014)C03. 